\title{
RAPID MRI analysis may speed up selection for tPA therapy
}

RAPID, an automated image-analysis program, can calculate stroke lesion volumes from diffusion-weighted and perfusion-weighted MRI (DWI and PWI) within $10 \mathrm{~min}$ and without requiring operator input. "These features make RAPID an attractive tool for patient selection in acute stroke trials," report Maarten Lansberg and colleagues in Stroke. Specifically, RAPID-generated images could be used to speed up and standardize the selection of patients for reperfusion therapy: "RAPID processing of a baseline MRI ... has the potential to determine whether a patient is a good candidate to receive treatment aimed at restoring perfusion to the ischemic brain," they conclude.

The team used RAPID to reprocess baseline MRI images obtained from 174 patients enrolled in two prospective studies of tissue plasminogen activator (tPA) therapy-DEFUSE and EPITHET. The program performed as well as manual calculations of lesion volumes in identifying which patients would show a favorable clinical response to $\mathrm{TPA}$, which was administered between $3 \mathrm{~h}$ and $6 \mathrm{~h}$ after stroke onset.
The patients expected to respond favorably to reperfusion therapy were those with a substantial difference between PWI and DWI lesion volumes, termed 'target mismatch', as calculated by RAPID. In this subgroup, reperfusion was strongly associated with a favorable clinical outcome and with attenuation of infarct growth following tPA treatment.

\section{4 ...RAPID-generated images} could be used to ... standardize the selection of patients for reperfusion therapy... 77

Lansberg et al. note that RAPID estimates of lesion size were consistently smaller than the manual estimates in the original study data, for both DWI and PWI scans. However, the two techniques resulted in similar proportions of patients being classified as having a target mismatch MRI profile.

Although the researchers state that RAPID MRI analysis does not provide an improved means of recognizing the patients likely to benefit from reperfusion therapy when compared with current manual techniques, they suggest that using this software might provide a standardized method of assessing MRI lesion size and profiles across different institutions. Manual methods for calculating stroke lesion volumes from MRI are timeconsuming and prone to interoperator variability; the resulting heterogeneity not only creates challenges for analyzing MRI data sets in multicenter clinical trials, but also impedes both comparisons of results from different studies and the development of definitive guidelines for MRI-based selection of patients for reperfusion therapy.

Lansberg et al. are currently working on new versions of RAPID that are optimized for establishing MRI profiles in the acute clinical setting, where factors such as motion artifacts can limit MRI quality.

Vanisha Parekh

Original article Lansberg, M. G. et al.

RAPID automated patient selection for reperfusion therapy: a pooled analysis of the Echoplanar Imaging Thrombolytic Evaluation Trial (EPITHET) and the Diffusion and Perfusion Imaging Evaluation for Understanding Stroke Evolution (DEFUSE) study. Stroke doi:10.1161/ STROKEAHA.110.609008 